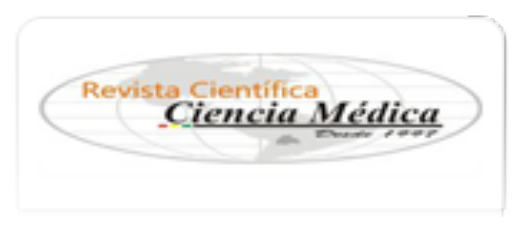

Revista Científica Ciencia Médica

ISSN: 1817-7433

ISSN: 2220-2234

revista_cienciamedica@hotmail.com

Universidad Mayor de San Simón

Bolivia

Camelo Rojas, Laura Vannesa; Piñeros Carranza, Gina Esperanza; Chaves Bazzan, Luzetty FOMENTO DE ALIMENTACIÓN LABORAL SALUDABLE EN AMÉRICA DEL SUR

Revista Científica Ciencia Médica, vol. 23, núm. 1, 2020, -Junio, pp. 61-68

Universidad Mayor de San Simón

Cochabamba, Bolivia

Disponible en: http://www.redalyc.org/articulo.oa?id=426064021009

Cómo citar el artículo

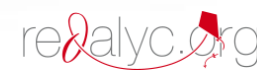

Número completo

Más información del artículo

Página de la revista en redalyc.org

Sistema de Información Científica Redalyc Red de Revistas Científicas de América Latina y el Caribe, España y Portugal Proyecto académico sin fines de lucro, desarrollado bajo la iniciativa de acceso abierto 


\title{
FOMENTO DE ALIMENTACIÓN LABORAL SALUDABLE EN AMÉRICA DEL SUR
}

HEALTHY EATING PROMOTION AT WORK

\begin{abstract}
Laura Vannesa Camelo Rojas', Gina Esperanza Piñeros Carranza', Luzetty Chaves Bazzani²
\end{abstract}

\section{RESUMEN}

La alimentación es un factor fundamental en la calidad de vida de los trabajadores, ya que aporta los nutrientes requeridos por el organismo para un funcionamiento apropiado. Teniendo en cuenta esto, se realizó una revisión documental de artículos científicos sobre la alimentación laboral en los países de Sur América a través de una búsqueda en las bases de datos Medline, Scielo y Ebsco en el periodo 2010 - 2019. Los artículos seleccionados fueron experiencias de programas de promoción de la alimentación saludable en el entorno laboral. En términos generales, se identificó cómo desde las acciones implementadas en el nivel individual, ambiental y organizacional aumentó el consumo de frutas y vegetales en la dieta de los trabajadores. Del mismo modo se evidencia que las experiencias de alimentación laboral saludable en América del Sur son limitadas desde el enfoque de la promoción de la salud.

\section{ABSTRACT}

Food is an important factor in the life quality of workers, since it provides the nutrients required by the body for proper functioning. Taking this into account, a documentary review of scientific articles on labor feeding in the countries of South America was carried out through a search in the Medline, Scielo and Ebsco databases, in the period 2010 - 2019. The articles selected were experiences of programs to promote healthy eating in the work environment. In general terms, it was identified how from the actions implemented at the individual, environmental and organizational level, the consumption of fruits and vegetables in the workers' diet increased. In the same way it is evident that the experiences of healthy work feeding in South America are limited from the health promotion approach.

Keywords: Health promotion, Employment, Healthy Diet, Occupational Health.

\section{INTRODUCCIÓN}

C omo lo plantean Martínez y Pedrón', la alimentación es un factor fundamental en el ciclo de vida de las personas, puesto que está ligada directamente con su desarrollo integral, su calidad de vida y el rendimiento, además de contribuir a la promoción de la salud y a la prevención de enfermedades. Desde el ámbito laboral, una inadecuada alimentación tiene un impacto negativo en los trabajadores, ya que conlleva a cansancio físico y mental, presentismo laboral, falta de motivación, entre otros aspectos. Según la Organización
Internacional del Trabajo $(\mathrm{OIT})^{2}$ la alimentación es uno de los componentes que condicionan el desarrollo físico, la salud y la productividad de las personas, ya que es la forma de aportar al cuerpo humano nutrientes y sustancias necesarias para mantener en estado óptimo la salud y la vida. Considerando los límites en las cantidades de azúcares, sal y en general las proporciones de los alimentos a consumir, se constituye en una fuente de nutrientes con sus correspondientes beneficios para el organismo. Según Mariño et $\mathrm{al}^{3}$, una alimentación
'Estudiante del Programa de Administración de Riesgos, Seguridad y Salud en el Trabajo. Semillero de Investigación TIKRAY. Universidad Militar Nueva Granada.

${ }^{2} \mathrm{Mg}$. Seguridad y Salud en el trabajo, Docente Universidad Militar Nueva Granada

Correspondencia a:

Laura Vannesa Camelo Rojas. Programa de Administración de Riesgos, Seguridad y Salud en el Trabajo. Universidad Militar Nueva Granada. Cra 11 No 101 - 80, Bogotá, Colombia. Correo electrónico: u0801441@ unimilitar.edu.co

Palabras clave: Promoción de la salud, Trabajo, Dieta saludable, Salud laboral

Keywords: Health promotion, Employment, Healthy Diet, Occupational Health.

Procedencia y arbitraje: no comisionado, sometido a arbitraje externo. Recibido para publicación: 20 de agosto 2019 Aceptado para publicación: 30 de mayo 2020

\section{Citar como:}

Camelo-Rojas LV, Piñeros-Carranza G, Chaves-Bazzani L. Fomento de alimentación laboral saludable en América del Sur. Rev Cient Cienc Med 2020; 23 (1): 61-68 
adecuada contribuye al fortalecimiento de la nutrición, siendo ésta el conjunto de procesos con los cuales el cuerpo humano, al recibir las sustancias contenidas en los alimentos las transforma, para después utilizarlas y convertirlas en defensas, con el fin de contribuir en los nutrientes necesarios que requiere el cuerpo humano y mantener un óptimo crecimiento. Según el autor, una buena alimentación debe estar equilibrada, con el aporte de cantidades adecuadas de los alimentos a consumir, debe ser completa y diversa con los nutrientes necesarios para que el organismo tenga un buen funcionamiento y con ello buena salud.

Rosales et $\mathrm{al}^{4}$, explican que la alimentación saludable proporciona todos los nutrientes esenciales y la energía que requiere cada persona para mantener una buena condición de salud. Esto se logra mezclando alimentos de manera equilibrada, que contribuyan al crecimiento y desarrollo de las capacidades físicas e intelectuales. La presencia de una importante variedad de enfermedades se relaciona con las características de los alimentos y hábitos en su consumo, como en el caso de las enfermedades cardiacas, la diabetes y la obesidad.

Bejarano y Díaz ${ }^{5}$ abordan la alimentación laboral con la visión de la OIT. Los autores señalan que desde 1953 ya se mencionaba la importancia de la alimentación en el entorno laboral, es decir, la alimentación ya se comenzaba a considerar como una estrategia de promoción y bienestar para la salud de los trabajadores. En el ambiente laboral, lograr una alimentación saludable cobra importancia desde las últimas décadas debido a que se evidenciaron efectos económicos negativos cuando muchos de los trabajadores disminuían su rendimiento laboral por alteraciones en su estado nutricional. Debido a estos hallazgos, se esperaría que la inversión en medidas de promoción de la salud en el espacio laboral, podría contribuir al disfrute de los derechos humanos fundamentales al tiempo que permitiría una reducción de costos. Estos autores señalan que Christopher Wanjek determina que los problemas nutricionales que tiene la población mundial impactan considerablemente en su capacidad tanto física como mental, disminuyendo de este modo la capacidad de trabajar, por lo tanto, es importante la aplicación de intervenciones en alimentación saludable debido a que mejora la calidad de vida y ayuda a disminuir el ausentismo laboral.

Retomando la iniciativa de la OIT, desde 1956 este organismo planteó en la Recomendación 102 la importancia de la alimentación en los lugares de trabajo en la cual se enfatizó en la necesidad de tener instalaciones adecuadas para facilitar la alimentación de los trabajadores OIT' ${ }^{6}$. Por otro lado, Lang et al ${ }^{7}$ implementaron para los trabajadores un apoyo, de tal modo que éstos efectuaran sus labores con técnicas saludables. Este programa se realizó basado en los antecedentes y logró ser visto desde diferentes perspectivas, también catalogando el estudio y beneficio de los mismos. El programa nacional de trabajo saludable (NHWP), fue elaborado para que los empleadores aprendan a implementar y aplicar sus propios programas enfocados en la salud y la productividad, demostrando, que cuando se les brinda capacitación y apoyo para establecer un ambiente saludable en el lugar de trabajo y una estrategia de planificación basada en datos se refleja un cambio positivo en los trabajadores.

En Chile, la $\mathrm{OIT}^{8}$ realizó un estudio con diversas empresas, del cual surgieron recomendaciones sobre la alimentación en los lugares de trabajo. Se afirma que, en el trabajo, la alimentación no debería ser considerada como un gasto sino como una inversión que minimiza los costos para el empleador, previniendo enfermedades crónicas, como la diabetes y aportando a la empresa debido a una mayor productividad.

Al considerar la relevancia de ésta temática para el desarrollo de la región, el objetivo de esta revisión es identificar las investigaciones realizadas en América del Sur y publicadas en revistas científicas, relacionadas con experiencias en la implementación de programas de alimentación saludable para los trabajadores.

\section{METODOLOGÍA}

Se realizó una revisión descriptiva que según Squires (1994), ofrece al lector "una puesta al día sobre conceptos útiles en áreas en constante evolución" (9). Las revisiones descriptivas son importantes ya que permiten tener una actualización sobre temas de interés.

Para el abordaje de los documentos, se 
realizó una revisión en las bases de datos Medline, Scielo y Ebsco. El área geográfica delimitada para la búsqueda de los artículos fue Sur América, con la búsqueda de artículos publicados en el periodo de 2010 a 2019 y con los descriptores "promoción de la salud", "trabajo" y "dieta saludable" en su título y resumen. Como criterios de inclusión se definieron los estudios que dieran cuenta de experiencias o puesta en marcha de programas de alimentación saludable en el ámbito laboral. Se excluyeron los estudios en los cuales se caracterizan los hábitos alimentarios, las condiciones de trabajo o las condiciones de salud de los trabajadores relacionadas con la alimentación. De acuerdo con la revisión realizada se identificaron 75 artículos, de los cuales siete presentaron experiencias sobre implementación de programas en el entorno laboral. Los demás artículos se excluyeron ya que se orientaban a caracterización de aspectos de salud relacionados con la alimentación en el trabajo, no contemplaban el entorno laboral o se limitaban a la descripción de condiciones de trabajo relacionadas con alimentación laboral.

\section{DESARROLLO}

Según Angulo et $\mathrm{al}^{10}$, la evidencia sobre intervenciones respecto a la alimentación saludable en los lugares de trabajo es escasa. Las dietas saludables en el entorno laboral tienen como fin promocionar la salud y prevenir enfermedades. Por medio de la educación, información sobre buenas conductas alimentarias, la implementación de programas de promoción de la salud en el entorno laboral proyecta una mejoría en la calidad de vida y salud de los trabajadores y con ello la productividad de la empresa crece notablemente, se obtiene como resultado la aplicación de un lugar saludable en el ambiente de trabajo, por lo que en algunas industrias reconocen estos factores como un enfoque potencial para influir en los trabajadores.

Complementando lo anterior y de acuerdo con Geaney et $\mathrm{al}^{11}$, es fundamental resaltar que la alimentación saludable se ha vuelto un factor de gran importancia en el transcurso de los años debido a que, si no se tiene en cuenta un entorno laboral saludable en las industrias, se ocasionan daños significativos tanto en sus trabajadores como en las finanzas de la empresa.

A partir de esta revisión se evidenció que, de los siete artículos identificados mencionados anteriormente, los países que reportaron estudios acerca de experiencias de alimentación laboral son Brasil, Paraguay (4 y 2 artículos respectivamente) y Chile (un artículo) (Tabla 1).

Programas y experiencias de alimentación saludable en Brasil:

Los cuatro artículos identificados en Brasil están conjuntamente relacionados, debido a que en la mayoría las empresas estaban registradas en el Programa de Alimentos para Trabajadores (PAT), que consiste en mejorar las condiciones de la alimentación en el trabajo, con el fin de obtener resultados positivos para la calidad de vida, el aumento de la productividad y la reducción de los accidentes laborales.

Pellegrino et $\mathrm{al}^{12}$, aplicaron un programa de promoción de la salud a 10 trabajadores del puerto de Río Grande, sur de Brasil, el programa tuvo una duración de 22 semanas, fundamentado en reuniones con el nutricionista cada semana, adoptando temas grupales tomando en consideración la implementación de los buenos hábitos alimenticios no solo con los miembros de la tripulación, sino también con sus familias para que tomaran conciencia y vieran la diferencia entre comer y alimentarse. Se evidencio que el trabajo central lo tenía el cocinero en la compra de alimentos más saludables y los menús más apropiados con una disminución considerable en las calorías, con esto los resultados obtenidos fueron una mejoría en la salud de los trabajadores y una mayor estabilidad de su peso.

A modo similar Franco et $\mathrm{al}^{13}$, evaluaron el impacto de las actividades que promueven el consumo de frutas y verduras en el lugar de trabajo con el programa de alimentación PAT. Luego de la intervención realizada (que incluyó información, cambios en la presentación de los alimentos, organización) se evidenció un incremento en el consumo de frutas y verduras.

Chaise y Santos ${ }^{14}$, evaluaron el conocimiento que tenían los gerentes administrativos y técnicos (nutricionistas) en ocho empresas sobre el programa mencionado. Se encontró que los encargados de este programa tienen conocimientos limitados a nivel de nutrición de los trabajadores.

Por su parte, Bandoni et al ${ }^{15}$, realizó un estudio experimental con 29 empresas que estaban 
Tabla 1. Experiencias documentadas sobre alimentación laboral en América del Sur

\begin{tabular}{|c|c|c|c|c|}
\hline $\mathbf{N}^{\circ}$ & PAís & AUTOR & NOMBRE DEL ARTÍCULO & FUENTE \\
\hline 1 & Brasil & $\begin{array}{l}\text { Amanda Silva Franco, Inés } \\
\text { Rugani Ribeiro de Castro } \\
\text { y Daisy Blumenberg Wo- } \\
\text { lkoff. }\end{array}$ & $\begin{array}{l}\text { Impact of the promotion of fruit and } \\
\text { vegetables on their consumption in } \\
\text { the workplace. }\end{array}$ & Rev Saúde Pública. 47(1) \\
\hline 2 & Brasil & $\begin{array}{l}\text { Lúcia Chaise Borjes y Ja- } \\
\text { naína Santos de Lima. }\end{array}$ & $\begin{array}{l}\text { Programa de Alimentação do Trabal- } \\
\text { hador: avaliando o conhecimento por } \\
\text { parte dos gestores administrativos e } \\
\text { técnicos. }\end{array}$ & $\begin{array}{l}\text { Demetra: alimentação, } \\
\text { nutrição \& saúde. } \\
\text { 9(1); 107-119 }\end{array}$ \\
\hline 3 & Brasil & $\begin{array}{l}\text { Daniel Henrique Bando- } \\
\text { ni, Flávio Sarno y Patricia } \\
\text { Constante Jaime. }\end{array}$ & $\begin{array}{l}\text { Impact of an intervention on the avai- } \\
\text { lability and consumption of fruits and } \\
\text { vegetables in the workplace. }\end{array}$ & $\begin{array}{l}\text { Public Health Nutrition. } \\
14(6), 975-981\end{array}$ \\
\hline 4 & Brasil & $\begin{array}{l}\text { Cristina Pellegrino Baena, } \\
\text { Ana Luísa Muccillo Baisch, } \\
\text { Tabajara Lucas Almeida, } \\
\text { Clarice De La Rocha, Ozéia } \\
\text { Simões Franco, Daniel Ol- } \\
\text { medo, Maria Cristina Flo- } \\
\text { res Soares. }\end{array}$ & $\begin{array}{l}\text { Impacto de un programa piloto de } \\
\text { promoción de la salud para trabajado- } \\
\text { res marítimos remolcadores }\end{array}$ & $\begin{array}{l}\text { Rev. bras. salud ocupa- } \\
\text { cional vol. } 36 \text { no. } 124\end{array}$ \\
\hline 5 & Paraguay & $\begin{array}{l}\text { Fabiana Paredes, Liz Ruiz y } \\
\text { Natalia González. }\end{array}$ & $\begin{array}{l}\text { Hábitos saludables y estado nutricio- } \\
\text { nal en el entorno laboral. }\end{array}$ & $\begin{array}{l}\text { Rev Chil Nutr. 45(2): } 119- \\
127\end{array}$ \\
\hline 6 & Paraguay & $\begin{array}{l}\text { Natalie Ríos, Margarita Sa- } \\
\text { mudio, Fabiana Paredes y } \\
\text { Fernando Vio. }\end{array}$ & $\begin{array}{l}\text { Efecto de una intervención educativa } \\
\text { nutricional en un entorno laboral. }\end{array}$ & $\begin{array}{l}\text { Archivos Latinoamerica- } \\
\text { nos de Nutrición. Tomo } \\
67, \text { N. }{ }^{\circ} 2\end{array}$ \\
\hline 7 & Chile & $\begin{array}{l}\text { Judith Salinasa, Lydia Le- } \\
\text { rab, Carmen Gloria Gonzá- } \\
\text { lezc, Fernando Vio. }\end{array}$ & $\begin{array}{l}\text { Evaluación de una intervención edu- } \\
\text { cativa nutricional en trabajadores de } \\
\text { la construcción para prevenir enfer- } \\
\text { medades crónicas no transmisibles en } \\
\text { Chile }\end{array}$ & $\begin{array}{l}\text { Rev. méd. Chile vol.144 } \\
\text { no. } 2\end{array}$ \\
\hline
\end{tabular}

Fuente. Elaboración propia a partir de la búsqueda documental para este estudio.

inscritas en el programa PAT. Se emplearon estrategias de cambio en el ambiente laboral, incluyendo: manuales, guías nutricionales para los administradores de la cafetería, talleres culinarios, distribución de materiales educativos orientados al consumo de frutas, verduras y en general alimentación balanceada. Se encontró que la puesta en marcha de las estrategias permitió establecer un ambiente de trabajo saludable, y generar principalmente en los trabajadores un mayor consumo de vegetales.

Programas y experiencias de alimentación saludable en Paraguay:

Paredes et $\mathrm{al}^{16}$, compararon el estado de nutrición y la rutina alimenticia de dos empresas de telefonía. En la empresa que se contaba con asesoría nutricional, espacios para actividad física orientada y re-educación alimentaria, se encontró que los trabajadores contaban con mejores niveles nutricionales, disminución en azúcares y aumento en el consumo de vegetales, frutas y lácteos.

Por su parte, Ríos et al ${ }^{17}$, exponen un estudio sobre una empresa de telefonía en donde, se implementó un programa de ambientes saludables. Se les proporcionó a los trabajadores intervención educativa nutricional con sesiones de consejería individual, acciones colectivas de apoyo, planificación del menú, mensajes de texto positivos y la puesta en marcha de un concurso de descenso de peso. Se evidenció una disminución en el consumo de alimentos y bebidas azucaradas, un mayor consumo en 
Tabla 2. Características y beneficios de la dieta Mediterránea

$\begin{aligned} & \text { CARACTERÍSTICAS DE LA DIETA MEDITE- } \\ & \text { RRANEA }\end{aligned}$
$\begin{array}{ll}\text { Comidas a base de vegetales, pequeñas } & \text { BENEFICIOS } \\ \text { cantidades de carne. } & \text {-Niveles de azúcar en sangre más estables. } \\ \text { Porciones de frutas, verduras frescas, nueces } & \text {-Aporte de hierro y vitamina E. } \\ \text { y legumbres. } & \text {-Reducción en el síndrome metabólico. } \\ \text { Alimentos con altas cantidades altas de } & \text {-Riesgo menor de desarrollar enfermeda } \\ \text { fibra } & \text { des cardíacas y de varios tipos } \\ \text { Consumo de pescado y otros mariscos. } & \text { de cáncer. } \\ \text { Aceite de oliva como la fuente principal de } & \\ \text { grasa empleada para preparar los alimentos. } & \\ \text { Preparaciones simples, sin salsas ni jugos de } & \\ \text { carne. } & \\ \text { Consumo de vino moderado (Excepto em- } \\ \text { barazo, propensión al alcoholismo, riesgo } \\ \text { de desarrollar cáncer de mama, otros). }\end{array}$
Fuente. Elaboración propia a partir de la búsqueda documental para este estudio.

vegetales, frutos secos y frutas.

\section{Alimentación laboral saludable en Chile:}

Desde el punto de vista de Martínez et al ${ }^{18}$, la dieta mediterránea consiste en el consumo de grandes porciones de vegetales, frutas, frutos secos y del mismo modo el consumo moderado de pescado y lácteos como los yogures y quesos, y en un bajo consumo de carnes y en el suministro de grasa poliinsaturada, que como señala Cabezas et a ${ }^{19}$ son grasas saludables que se encuentran en aceites vegetales. Estudios epidemiológicos han determinado que la ingesta de esta dieta mediterránea se asocia a la prevención de enfermedades cardiovasculares, incluyendo tipos de cáncer. Algunos beneficios y demás características de la dieta mediterránea se muestran en la tabla 2.

Salinas et $\mathrm{a}^{20}$, explica el programa que uso en su estudio sobre una intervención educativa nutricional por lo que hay similitud con la dieta mediterránea debido a la ingesta de los alimentos suministrados en trabajadores de construcción, que consistía en tres sesiones: consejería individual, donde se hacían recomendaciones a cada trabajador para que aprendieran a moderar el consumo de azucares, grasas, pan y cantidad de sal en sus comidas, motivándolos a que día a día consumieran cinco porciones de frutas y verduras, aumentando el consumo de legumbres, entregando material educativo sobre alimentación saludable.
La segunda sesión eran talleres educativos grupales, caracterizados por el manejo y la importancia en los cambios para una buena nutrición y la tercera sesión consistía en consejerías grupales, tomando como temas principales el agua como parte fundamental para el organismo, en lugar de las bebidas que contenían azúcar. Adicionalmente se hicieron campañas con volantes y posters sobre la buena alimentación. Como resultados en un periodo corto se evidencio que los trabajadores habían disminuido en el consumo de calorías y habían aumentado notablemente la ingesta de grandes porciones de pescado, frutas $y$ verduras.

En los programas encontrados se identificaron acciones que se categorizaron en tres componentes: Intervención individual, intervención sobre el ambiente de trabajo y finalmente, intervención sobre la organización, como se muestra en la tabla 3.

\section{DISCUSIÓN}

En este estudio se evidenciaron limitadas experiencias investigativas documentadas respecto a la promoción de la alimentación saludable en el entorno laboral. Es importante destacar, la iniciativa creciente en las últimas décadas por parte del sector público, privado y la academia, en esta temática tan relevante 
Tabla 3. Categorías emergentes del estudio

\begin{tabular}{|c|c|}
\hline \multicolumn{2}{|c|}{ PROGRAMAS DE ALIMENTACIÓN LABORAL EN SUR AMÉRICA } \\
\hline COMPONENTE & DESCRIPCIÓN \\
\hline Intervención individual & $\begin{array}{l}\text { - Educación dirigida a trabajadores. } \\
\text { - Asesoría nutricional: Reeducación alimentaria } \\
\text { individualizada, planes de alimentación adapta- } \\
\text { dos a la realidad y gustos del paciente. } \\
\text { - Consultas de consejería individual. } \\
\text { - Concurso para bajar de peso. }\end{array}$ \\
\hline Intervención sobre al ambiente de trabajo & $\begin{array}{l}\text { - Adecuación de ambiente en comedores. } \\
\text { - Disponibilidad de frutas y hortalizas para con- } \\
\text { sumo a demanda. } \\
\text { - Planeación de menú. } \\
\text { - Estrategias de presentación de los alimentos y } \\
\text { motivación para incentivar el consumo de frutas } \\
\text { y vegetales. } \\
\text { - Ambientes saludables en general: Lugar des- } \\
\text { tinado a la práctica de actividad física durante la } \\
\text { jornada laboral y áreas libres de Tabaco. }\end{array}$ \\
\hline Intervención sobre la organización & $\begin{array}{l}\text { - Sobre la empresa: Gerencia y en general gesto- } \\
\text { res de la empresa, intervenciones administrativas, } \\
\text { organización del trabajo. } \\
\text { - Sobre el proveedor: Servicio de alimentación } \\
\text { con algunas opciones recomendadas por profe- } \\
\text { sionales, nutricionistas. } \\
\text { - Acciones colectivas de apoyo. }\end{array}$ \\
\hline
\end{tabular}

Fuente. Elaboración propia a partir de la búsqueda documental para este estudio.

para los trabajadores de América del Sur. Sin embargo, considerando los perfiles de morbilidad y mortalidad relacionados con enfermedades crónicas en la región, emerge la necesidad de impulsar estas iniciativas con un mayor vigor, para que logre impactar positivamente en la salud y bienestar de los trabajadores y de la población en general.

Aunque en la región se encontraron diversas publicaciones en torno al tema, se trata principalmente de reflexiones 0 caracterizaciones de condiciones de salud y trabajo relacionadas con el consumo de alimentos por parte de los trabajadores. Por otra parte, se encontraron artículos en los cuales, aunque no se documenta una experiencia investigativa formal respecto a la puesta en marcha de un programa, si plantea iniciativas relevantes. Es el caso del planteamiento de Llamas $^{21}$, con el programa creado por la Fundación Colombiana del Corazón, denominado; "Corazones responsables", creado para que las empresas tuvieran la oportunidad de mejorar la calidad de vida de sus trabajadores y preservar su salud. El autor explica que una organización saludable debe estar fundamentada en la integración de todos los trabajadores y que, con ello, la alimentación laboral saludable se promueve para conservar la salud y el bienestar de los trabajadores, no solo la salud física sino también la salud mental, ya que estos factores en buen estado favorecen la disminución el presentismo y ausentismo laboral. Se plantea a través de este programa, una alternativa de certificación a las empresas que cumplan con determinados criterios que incluyen entre otros aspectos, lo relacionado con la alimentación saludable.

Seevidencia con las experiencias identificadas que paulatinamente los programas en la 
región adquieren una mayor integralidad. La promoción de la salud deja de ser una serie de charlas aisladas a los trabajadores, para convertirse en procesos de múltiples componentes. La alimentación saludable, acompañada de actividad física, prevención de consumo de tabaco todo enlazado a las estrategias y diversos componentes planteados desde el área de seguridad y salud en el trabajo, permite favorecer las condiciones de salud en las organizaciones. Un ejemplo claro de esto es el impacto de la promoción del consumo de frutas y hortalizas en las experiencias documentadas en Brasil. Los programas incluían múltiples componentes y las intervenciones incluían las de tipo individual, sobre el ambiente de trabajo y sobre la organización. Es importante considerar estos tres factores, dado que se puede lograr un efecto directo tanto para el bienestar del trabajador, como para el beneficio de la empresa.

Las experiencias identificadas incluyeron cambios en el ambiente laboral, con lo cual se generaron condiciones para favorecer la salud y bienestar de los trabajadores. Es importante resaltar como varias de estas empresas, al generar un nuevo conocimiento, cambiaron la calidad y estilo de vida, como señalan Ríos et al ${ }^{17}$ en cuanto a los efectos de una intervención educativa nutricional en un entorno laboral.

Es importante una alimentación balanceada, puesto que es responsabilidad de los empleadores con los trabajadores. La Organización Mundial de la Salud y la Organización Internacional del Trabajo desde hace varias décadas han enfatizado en la necesidad de realizar todos los esfuerzos en torno a la promoción de la salud. Se requiere una mayor iniciativa de los gobiernos y de los empresarios, para lograr un mayor compromiso con el diseño y seguimiento a programas de promoción en las empresas y generar iniciativas para que las empresas opten por estos programas como prioridad. Es necesario promover el interés por parte de las empresas y de los trabajadores. Una política que contemple la educación alimentaria y el acceso a alimentos contribuiría a mejorar la salud y prevenir enfermedades. Finalmente es también un gran reto para el sector educativo. Desde la academia, a partir de la identificación de las necesidades, es posible aportar al desarrollo de propuestas de solución para beneficio de la salud de los trabajadores

\section{CONCLUSIÓN}

A partir de la revisión realizada, se identificaron siete artículos publicados en revistas científicas, con investigaciones realizadas en América del Sur sobre la implementación de programas de alimentación saludable para los trabajadores, en el período 2010 a 2019. Los países que reportaron los estudios fueron Brasil, Paraguay y Chile.

Se destaca que los programas de alimentación laboral son más efectivos si incluyen acciones en diversos componentes: Individual, ambiente de trabajo y organización. Con la puesta en marcha de los programas que incluyen acciones desde los diferentes componentes, se evidenció que el consumo de frutas y vegetales y en general de alimentos saludables se aumenta entre los trabajadores.

Un entorno laboral saludable incorporado a una empresa incide sobre hábitos de alimentación y salud, siendo considerado como un factor primordial en la calidad de vida de los trabajadores.

Se evidencian muy pocas las experiencias de alimentación laboral saludable desde el enfoque de la promoción de la salud que se han documentado a partir de procesos de investigación en América del Sur. Es necesario evaluar los programas que se están llevando a cabo en diversas organizaciones y publicar sus resultados, ya que al compartir experiencias será posible mejorar las intervenciones y por lo tanto la salud de los trabajadores de la región. 


\section{REFERENCIAS}

1. Martínez AB, Pedrón C. Conceptos Básicos en Alimentación [Internet]. 2017 [citado 2019 Oct 27] Disponible en: https://www.seghnp.org/sites/default/ files/2017-06/conceptos-alimentacion.pdf

2. Organización Internacional del Trabajo. Alimentación Sana. [Internet]. 2018 [citado 2019 Oct 28]. Disponible en: https://www.who.int/es/news-room/fact-sheets/detail/ healthy-diet

3. Mariño A, Núñez M, Gámez Al. Alimentación saludable. [Internet]. 2015 [citado 2019 Oct 28]. Disponible en: https:// www.medigraphic.com/pdfs/actamedica/acm-2016/ acm161e.pdf

4. Rosales Y, Peralta L, Yaulema L, Pallo J, Orozco D, Caiza $V$, Parreño A, Barragán V, Ríos P. Alimentación saludable en docentes. RCMGI [Internet]. 2017 [citado 2019 Oct 29]; 33(1):115-128. Disponible en: https://www.medigraphic. com/pdfs/revcubmedgenint/cmi-2017/cmi171j.pdf

5. Bejarano JJ, Díaz M. Alimentación laboral una estrategia para la promoción de la salud del trabajador. Rev Fac Med. [Internet]. 2012 [citado 2019 Oct 29]; 60 (Supl): S87-97. Disponible en: http://www.scielo.org.co/pdf/ rfmun/v60s7/v60s7a09.pdf

6. Organización Internacional del Trabajo. Santiago, Chile. [Internet]. 2015 [citado 2019 Oct 29]. Disponible en: https:// www.ilo.org/santiago/sala-de-prensa/WCMS_365638/ lang--es/index.htm

7. Lang J, Cluff, L, Payne J, Matson D, Hampton J. The Centers for Disease Control and Prevention: Findings From The National Healthy Worksite Program. Occup Environ Med [Internet]. 2017 [citado 2020 Abr 6]; 59(7): 631-641. Disponible en: https://www.ncbi.n/m.nih.gov/pmc/articles/ PMC5708873/

8. Organización Internacional del Trabajo. Un enfoque integral para mejorar la alimentación y nutrición en el trabajo. Estudio en empresas chilenas y recomendaciones adaptadas. Santiago, Chile. [Internet]. 2012 [citado 2019 Oct 29]; Disponible en: https://www.ilo.org/wcmsp5/groups/ public/---americas/---ro-lima/---sro-santiago/documents/ publication/wCMs_201138.pdf

9. Vera O. CÓMO ESCRIBIR ARTÍCULOS DE REVISIÓN. Rev. Méd. La Paz [Internet]. 2009 [citado 2020 My 24]; 15 (1): 63-69. Disponible en: http://www.scielo. org.bo/scielo.php?script=sci_arttext\&pid=S172689582009000100010\&lng=es.

10. Angulo N, Aparicio M, Marco M, Sanjuán V. Nutrición en el trabajo y actividades de promoción de la salud: revisión sistemática. Med. seg. trab. [Internet]. 2018 [citado 2019 Oct 31]; 64 (251): 200-216. Disponible en: http:// scielo.isciii.es/scielo.php?script=sci_arttext\&pid=S0465546X2018000200200\&lng=es.

11. Geaney F, Kelly C, Scotto J, Harrington J, Fitzgerald A, Greiner B, Perry I. El efecto de las complejas intervenciones dietéticas en el lugar de trabajo sobre la ingesta alimentaria de los empleados, el conocimiento nutricional y el estado de salud: un ensayo controlado por conglomerados. Elsevier. [Internet]. 2016 [citado 2019 Oct 31]; 89: 76-83. Disponible en: https://WwW-sciencedirect-com.ezproxy.umng.edu.co/ science/article/pii/S0091743516300822

12. Pellegrino $B C$, Muccillo $A L$, Almeida $T L$, De La Rocha $C$, Franco OS. Olmedo D, Flores MC. Impacto de un programa piloto de promoción de la salud para trabajadores marítimos remolcadores. Rev. bras. saúde ocup. [Internet]. 2011

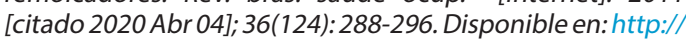
www.scielo.br/scielo.php?script=sci_arttext\&pid=S030376572011000200013\&lng=en. https://doi.org/10.1590/ S0303-76572011000200013.

13. Silva A, Ribeiro IR, Blumenberg D. Impact of the promotion of fruit and vegetables on their consumption in the workplace. Rev Saú Púb. [Internet]. 2013 [citado 2019 Oct 31]; 47(1). Disponible en: http://www.scielo.br/pdf/rsp/ v47n1/en_05.pdf

14. Chaise L, Santos J. Programa de Alimentação do Trabalhador: avaliando o conhecimento por parte dos gestores administrativos e técnicos. Demetra: alimentação, nutrição \& saúde, [Internet]. 2014 [citado 2019 Oct 31]; 9(1); 107-119. Disponible en: https://www.e-publicacoes.uerj.br/ index.php/demetra/article/viewFile/6643/8818

15. Bandoni DH, Sarno F, Constante P. Impact of an intervention on the availability and consumption of fruits and vegetables in the workplace. Public Health Nutrition, [Internet]. 2010 [citado 2019 Oct 31]; 14(6), 975-981. Disponible en: https://www.cambridge. org/core/services/aop-cambridge-core/content/ view/3F8F2DCF5788397C9B95FF67A15A2AA8/ S1368980010003460a.pdf/impact of an intervention on_the_availability_and_consumption_of_fruits_and_ vegetables_in_the_workplace.pd

16. Paredes F, Ruiz L, González N. Hábitos saludables y estado nutricional en el entorno laboral. Rev Chil Nutr. [Internet]. 2018 [citado 2019 Oct 31]; 45(2): 119127. Disponible en: https://scielo.conicyt.cl/pdf/rchnut/ v45n2/0716-1549-rchnut-45-02-0119.pdf

17. Ríos N, Samudio M, Paredes F, Vio F. Efecto de una intervención educativa nutricional en un entorno laboral. Archivos Latinoamericanos de Nutrición, [Internet]. 2017 [citado 2019 Oct 31]; Tomo 67, N. ${ }^{\circ}$ 2. Disponible en: https:// wWw.alanrevista.org/ediciones/2017/2/art-9/

18. Martínez MA, Gea A, Ruiz M. The Mediterranean Diet and Cardiovascular Health. Circ Res. [Internet]. 2019 [citado 2020 Abr 5]; 124(5): 779-798. Disponible en: https://www. ahajournals.org/doi/pdf/10.1161/CIRCRESAHA.118.313348

19. Cabezas CC, Hernández BC, Vargas M, Aceites y grasas: efectos en la salud y regulación mundial. Rev FaC Med. [Internet]. 2016 [citado 2019 Oct 31]; 64 (4): 7618. Disponible en: http://www.scielo.org.co/pdf/rfmun/ v64n4/0120-0011-rfmun-64-04-00761.pdf

20. Salinas J, Lera L, González CG, Vio F. Evaluación de una intervención educativa nutricional en trabajadores de la construcción para prevenir enfermedades crónicas no transmisibles en Chile. Rev. méd. Chile [Internet]. 2016 [citado 2020 Abr 06]; 144(2): 194-201. Disponible en: https:// scielo.conicyt.cl/scielo.php?script=sci_arttext\&pid=S003498872016000200008\&lng=es. http://dx.doi.org/10.4067/ S0034-98872016000200008.

21. Llamas A. Promoción de hábitos saludables en el trabajo para el bienestar de la población trabajadora. Rev Colomb Cardiol. [Internet]. 2014 [citado 2019 Oct 31]; 21(4):199-201. Disponible en: http://www.scielo.org.co/ pdf/rcca/v21n4/v21n4a01.pdf 